\title{
Was bringt die Lebensende-Forschung für die Ethik in der Medizin?
}

\author{
Markus Zimmermann-Acklin
}

Online publiziert: 22. Dezember 2013

(C) Springer-Verlag Berlin Heidelberg 2013

Spätestens dann, wenn ein dreibändiges Handbuch im Umfang von über 1200 Seiten erscheint, lässt sich die enorme Aufmerksamkeit, mit der heute das Sterben bedacht wird, nicht mehr übersehen [1]. Die Wahrnehmung oder „Entdeckung des Sterbens“ (Ursula Streckeisen) als einer eigenständigen Lebensphase ist von unterschiedlichen Deutungen, Idealen, aber auch Ängsten geprägt. Verursacht wird sie durch gesellschaftliche und institutionelle Veränderungen wie die demographische Entwicklung, das Sterben vieler Menschen in sehr hohem Alter, die Entstehung neuer Institutionen wie Hospize, Palliative Care-Einrichtungen, spezialisierte Pflegeheime, aber auch von Patientenverfügungen, rechtlichen Neuregelungen der Lebensende-Entscheidungen und nicht zuletzt von Sterbehilfeorganisationen. Meist steht nicht mehr der Kampf gegen den nahenden Tod, sondern die Sicherstellung eines ,guten Sterbens“ im Zentrum der Aufmerksamkeit.

Bereits dieser Versuch, komplexe gesellschaftliche Vorgänge in Bezug auf das Lebensende in wenigen Worten anzudeuten, indiziert, wie weitgehend hier ethisch-moralische Aspekte berührt werden. Das ist wenig erstaunlich, gehörte das Lebensende doch bereits zu den ersten großen Themen der in den 1960er Jahren neu entstandenen biomedizinischen Ethik. In den letzten Jahren ist die Einsicht gewachsen, dass die ethischen Debatten nur dann weiterführen, wenn auf der Basis gründlicher Sachkenntnis die richtigen Fragen gestellt und diskutiert werden. Wissen wir eigentlich, was sich im Bereich des Lebensendes gegenwärtig verändert? Werden in der Medizinethik die Herausforderungen aufgegriffen, welche Sterbende, deren Angehörige, Behandlungsteams, das Krankenhausmanagement oder auch die Gesundheitspolitik tatsächlich beschäftigen?

Die Erkundung dieser Herausforderungen ist in der Schweiz seit letztem Jahr Thema eines Nationalen Forschungsprogramms. ${ }^{1}$ In insgesamt 33 Einzelprojekten und in vier

${ }^{1}$ Nationales Forschungsprogramm NFP 67 “Lebensende”, www.nfp67.ch. Zugegriffen: 13. Dez. 2013.

PD Dr. theol M. Zimmermann-Acklin $(\bowtie)$

Departement für Moraltheologie und Ethik, Universität Fribourg,

Avenue de l'Europe 20, 1700 Fribourg, Schweiz

E-Mail: markus.zimmermann@unifr.ch 
Bereichen (1. Sterbeverläufe und Versorgung, 2. Entscheidungen und Einstellungen, 3. Regelungen und Handlungsvorschläge, 4. Leitbilder und Ideale) wird aus Sicht unterschiedlicher Disziplinen der Frage nachgegangen, was sich im Bereich des Lebensendes in der Schweiz heute verändert. Die Forschungsdauer beträgt fünf Jahre, das Programm wird mit einem Synthesebericht zuhanden des Schweizer Bundesrats abgeschlossen.

Inwieweit kann die Ethik in der Medizin von einem solchem Programm profitieren? Ich möchte diese Frage mit Hilfe Daniel Callahans beantworten, der vier Hauptbereiche der biomedizinischen Ethik, nämlich die theoretische, klinische, politisch-regulatorische und kulturelle Bioethik voneinander abgegrenzt hat ([2], S. 281).

Mit Blick auf die theoretische Medizinethik, den Versuchen, zentrale Begriffe und Begründungskonzepte zu klären, werden im Forschungsprogramm beispielsweise Projekte durchgeführt, welche das Verständnis der Autonomie im Bereich der Suizidhilfe bzw. das Konzept der Urteilsfähigkeit und deren Bestimmbarkeit bei Sterbenden erkunden. Die Medizinethik selbst ist insofern auch Teil des Programms. Daneben profitiert die klinische Medizinethik, die sich mit den alltäglichen Herausforderungen der Entscheidungsfindung befasst, auf mannigfaltige Weise: Beispielsweise wird im Rahmen eines Projekts die so genannte EURELD-Studie [3] zu Entscheidungen am Lebensende in der Schweiz wiederholt, daneben Entscheidungen und deren Hintergründe bei sterbenden Neugeborenen erkundet, es wird danach gefragt, gemäß welcher Kriterien Patienten in ihrer letzten Lebensphase auf eine Intensivstation verlegt werden oder auch, wie Sterbewünsche bei Menschen am Lebensende tiefer zu verstehen sind. Daneben werden zwei Interventionsstudien zum Bereich einer rechtzeitig einsetzenden Palliative Care durchgeführt. Mit Blick auf die politisch-regulatorische Medizinethik werden im Rahmen zweier juristischer Projekte bestehende Richtlinien und gesetzliche Regelungen erforscht und nach Verbesserungen gefragt. Andere Projekte erkunden die Lebensende-Versorgung marginalisierter Gruppen wie Menschen mit Behinderungen oder Patienten im Strafvollzug. In zwei Projekten werden die Bedürfnisse pflegender Angehöriger aufgegriffen, in anderen die hohen Kosten am Lebensende. Schließlich wird die kulturelle Medizinethik an Ergebnissen anknüpfen können, welche Hintergrundtheorien, Ideale und soziale Kontexte betreffen, wenn beispielsweise Sinn- oder Spiritualitätsbedürfnisse oder der Einfluss alternativer Religiosität auf das Sterben beleuchtet werden.

Was kann die Lebensende-Forschung für die Medizinethik erbringen? Einerseits ist die Ethik in der Medizin selbst Teil des größeren Ganzen, andererseits kann sie von den Ergebnissen unterschiedlichster Projekte im Sinne einer vertieften Kenntnis der Realität profitieren. Die interdisziplinäre Vernetzung der Medizinethik mit anderen Bereichen der Lebensende-Forschung bietet von daher gute Gelegenheit zur weiteren Etablierung der Ethik in der Medizin.

\section{Literatur}

1. Anderheiden M, Eckart WU (Hrsg) (2012) Handbuch Sterben und Menschenwürde, Bd. 1-3. De Gruyter, Berlin

2. Callahan D (2003) Art. „Bioethics“. In: Post SG (Hrsg) Encyclopedia of bioethics, 3. Aufl., Bd. 1. Macmillan Reference USA, New York, S 278-287

3. van der Heide A, Deliens L, Faisst K et al (2003) End-of-life decision-making in six European countries: descriptive study. The Lancet 362:345-350 